\title{
Erratum to: The mass-gap in Quantum Chromodynamics
}

\author{
S.A. Larin $^{1}$ \\ ${ }^{1}$ Institute for Nuclear Research of the Russian Academy of Sciences, $60^{\text {th }}$ October Anniversary Prospect 7a, Moscow 117312, Russia
}

Original article:

EPJ Web Conf.

Volume 125, 04001 (2016)

The 19th International Seminar on High Energy Physics (QUARKS-2016)

DOI: $10.1051 /$ epjconf/201612504001

There is a misprint in Eq. (8):

one should replace $\sqrt{3}$ by 3 . 